Voix et Images

\title{
Un livre de tête et de coeur : Philippe Haeck, la table d'écriture. Poétique et modernité
}

\section{Jacques Pelletier}

Volume 11, numéro 1, automne 1985

Naïm Kattan

URI : https://id.erudit.org/iderudit/200541ar

DOI : https://doi.org/10.7202/200541ar

Aller au sommaire du numéro

Éditeur(s)

Université du Québec à Montréal

ISSN

0318-9201 (imprimé)

1705-933X (numérique)

Découvrir la revue

Citer cet article

Pelletier, J. (1985). Un livre de tête et de coeur : Philippe Haeck, la table d'écriture. Poétique et modernité. Voix et Images, 11(1), 104-107.

https://doi.org/10.7202/200541ar d'utilisation que vous pouvez consulter en ligne.

https://apropos.erudit.org/fr/usagers/politique-dutilisation/ 


\title{
Essai
}

\section{Un livre de tête et de coeur: Philippe Haeck, la table d'écriture. Poétique et modernité,}

\author{
par Jacques Pelletier, Université du Québec à Montréal
}

Avec la Table d'écriture', Haeck s'affirme comme un grand essayiste. Ce qui n'était pas le cas avec l'Action restreinte. De la littérature (1975), ouvrage de polémiste ni même avec Naissances. De l'écriture québécoise (1979), production de thèsard original sans doute, mais thèsard tout de même. Ici il s'agit de tout autre chose: d'une réflexion libre, ouverte, créatrice sur la littérature québécoise contemporaine.

Le titre de l'ouvrage, comme toujours chez Haeck pour qui il s'agit d'un élément capital du texte, est on ne peut plus significatif. Ici il comporte deux éléments. La table d'écriture d'abord comme on pourrait dire la table du cordonnier, du menuisier, bref de l'artisan, de celui qui en l'occurrence produit, fabrique de l'écriture. Qui lit, mais ne se contente pas de lire. Qui réfléchit sur (et à partir de) ce qu’il lit, qui écrit, qui crée. Qui ne se contente pas de dégager la signification de textes-objets mais qui est transformé par eux, regarde le monde autrement après leur lecture et rend compte de sa nouvelle façon de voir les choses. Les textes comme points d'appui, supports d'une réflexion qui se déploie librement, qui produit du nouveau. Du commentaire, de la critique, oui, mais plus que cela: de la création.

Poétique et modernité ensuite, délimitation d'un objet donc: les textes qui, par leur caractère innovateur, ont transformé (transforment) la littérature et notre façon de voir le monde et choix d'une perspective, celle de considérer les textes dans ce qu'ils mettent en jeu, dans leurs rapports à l'existence: non pas purs objets de savoir livresque mais instruments d'appropriation du réel qui peuvent nous aider à voir plus clair dans nos vies. Ce n'est donc pas en savant à la recherche de lois, en poéticien qu'Haeck travaille sur les textes mais en homme de chair et d'os qui cherche à donner un sens à sa vie et à celle des autres.

Cet homme est un enseignant au niveau collégial, qui travaille donc avec des adolescents qui n'ont à priori guère d'intérêt pour la littérature qui leur semble coupée de la vie. Son objectif: leur faire aimer la littérature en leur montrant par son exemple (comment elle affecte sa vie) qu'elle peut être source de connaissance et de plaisir. D'où sa méfiance du traitement universitaire de la littérature comme objet de savoir auquel on applique des grilles pré-établies, qui font écran à une prise de connaissance directe des textes. D'où sa préoccupation pour la création: enseigner, c'est apprendre à écrire en montrant, en expliquant comment les écrivains de la modernité ont fait (font). Faire de la lecture et de l'écriture une seule et même expérience. C'est l'objectif de son enseignement, c'est aussi celui de son livre.

Sa méthode? D'abord ce qu'elle n'est pas: Mon travail a peu à voir avec les descriptions scientistes qu'on exécute dans les universités, qui étant souvent un plaquage stérile de grilles (dé)codantes, ne sont en rien critiques 
(p. 17). Ensuite ce qu'elle est: La critique telle que je l'entends s'occupe plus de philosophie, de lutte idéologique et de théorie poétique que des notions linguistiques, rhétoriques, sémiotiques, sociologiques, psychanalytiques. Pas de critique sans position éthique, sans questionnement des valeurs, sans savoir qui ne soil un geste vers quelqu'un (p. 17).

Rejet donc des démarches universitaires classiques, des discours se voulant scientifiques au profit d'une lecture politique au sens large du terme, considérant les textes en lien avec les individus et le procès historique dans lequel ils sont engagés. Critique faite à la lumière des enseignements de Freud, Marx, Nietzsche considérés non pas comme fournisseurs de modèles, de grilles à appliquer mais comme sources d'inspiration, penseurs originaux chacun dans leur domaine, les ayant renouvelés en prenant les problèmes à la racine.

Critique engagée aussi visant à exposer les enjeux (littéraires, culturels, sociaux) des textes, les considérant donc comme des pratiques transformatrices, produisant des effets sur le réel, et d'abord sur soi: d'où le souci de lire à partir de ce qu'on est: Je lis avec toute ma biographie, je ne peux y échapper - ce que je retiens d'un texte n'est que ce qui ébranle mon identité (p. 244). Critique enfin fondée sur ce que Haeck appelle l'improvisation, c'est-à-dire sur la constitution d'un discours d'accompagnement qui, tout en s'appuyant sur les textes, les dépasse de quelque manière et les intègre dans une réflexion autonome. En pratique cela se traduit par de brèves notes, improvisations, intuitions, rapides esquisses (p. 15). Rien de plus éloigné en somme des démonstrations rigoureuses en trois temps (thèse, antithèse, synthèse: le modèle est bien connu) des travaux académiques. Des notes plutôt, écrites au fil des lectures et des jours, pour éclairer sa route, trouver son chemin.

Les objets? Les poètes québécois de la modernité bien sûr qui, pour Haeck, ne commence pas avec La barre du jour mais avec Jean-Aubert Loranger et Saint-Denis Garneau, se poursuit avec les automatistes (PaulMarie Lapointe, Roland Giguère) au tournant des années 1950, se manifeste chez les écrivains influencés par la contre-culture américaine des années 1960 (Lucien Francoeur, Louis Geoffroy, Denis Vanier) et trouve son aboutissement dans le formalisme des écrivains - certains, pas tous - publiés à La barre du jour et aux Herbes rouges. La modernité, dans cette perspective, ne saurait être assimilée au travail textuel des formalistes des années 1970 pour lesquels Haeck éprouve des réserves, notamment à l'endroit de leur chef de file, Nicole Brossard, dont l'écriture froide et elliptique du début des années 1970 ne lui plaît guère alors que ses productions récentes - inspirées par le féminisme, dans lesquelles un je s'exprime librement - nomment l'utopie (p. 170) et par là renouent avec la vie. Ces réserves iront en s'amplifiant et aboutiront à une rupture avec les Herbes rouges en 1977: $s i$ j'avais été d'accord avec les écritures souvent difficiles des Herbes rouges c'est que je savais inévitable cette étape où une nouvelle génération cherche ses formes - sens mais je commençais à trouver que le temps de l'expérimentation formelle avait suffisamment duré: il fallait dépasser tous ces vers tronqués; toutes ces phrases elliptiques, qui commençaient à n'être plus qu'une marque de modernisme, ne correspondant plus à une marche mais à un piétinement (p. 105). 
Dans cet ordre d'idées, il introduit une distinction pertinente entre ce qu'il appelle le style formaliste qui pratique des transformations linguistiques et le style historique qui pratique des interventions idéologiques et manifeste sa préférence pour le style historique à condition qu'il ne néglige pas le travail du style et qu'il opère sur le sens à partir de la psychanalyse et du marxisme. On comprendra aisément, ceci rappelé, son admiration pour les écritures de Patrick Straram - écrivain trop négligé par la critique dont il trace un admirable portrait - qui a su intégrer les apports de Marx et de Nietzsche, la pratique et la théorie, le rythme et l'analyse, la création et la critique. De sa propre pratique poétique, Haeck ne parle guère, mais on imagine sans mal qu'il la situe dans ce courant historique.

Autres objets étudiés: des romans, quelques-uns seulement toutefois de manière approfondie: l'Amélanchier et le Ciel de Québec, ce livre de la fondation fantastique, cette mythologie des pays québécois comme disait Victor-Lévy Beaulieu dont Blanche forcée est retenu en tant que roman psychanalytique: l'analyste Job J. enferme sa cliente dans la ruse de son discours: il ne reste plus à la cliente qu'à sortir pour ne pas mourir étouffée par le discours (p. 196): elle en sort en effet de la façon dramatique que l'on sait, en s'ouvrant les veines; l'interprétation ne s'arrête pas à ce niveau cependant et c'est ce qui fait son intérêt: elle va plus loin débouchant sur la signification sociale du roman dont le héros est le reflet de l'intellectuel petitbourgeois des années soixante-dix, intellectuel beau-parleur pour qui la psychanalyse devient le terrain idéal pour faire valoir sa parole (p. 197). De même dans son étude de l'Enfant dans le grenier de Julien Bigras, Haeck recourt à la psychanalyse - s'agissant encore une fois d'un roman psychanalytique, cela va de soi - mais il utilise aussi la critique que l'on peut faire de celle-ci, de ses limites lorsqu'elle se borne à une théorie du sujet extrait de son ancrage historique. $\bar{A}$ nouveau son analyse contient des remarques fines et pertinentes, ouvrant des percées qui suscitent la réflexion et appellent à une remise en question des discours reçus, y compris celui de la psychanalyse.

Au centre de cette démarche critique: un témoignage qui rappelle clairement que celui qui parle est un sujet historiquement situé: Comment je suis devenu écrivant dans les années soixante-dix. Écrivant, non écrivain, notonsle: homme d'écriture de fiction, mais aussi de critique, homme de parole également, enseignant, militant syndical et politique se définissant volontiers comme communiste: écrivant, non écrivain mineur, au contraire écrivain agissant sur plusieurs terrains, écrivant au sein d'une conjoncture traversée par trois courants culturels d'importance: la contre-culture, l'écriture réflexive (le formalisme), l'écriture des femmes. La contre-culture, condamnée par l'auteur à l'époque de Chroniques (1975-1977) fait l'objet au début des années 1980 d'une ré-évaluation positive, ayant le mérite de rappeler que nous sommes d'abord américains (p. 121). L'écriture réflexive, chez ses meilleurs représentants, a donné des résultats intéressants. Et l'écriture des femmes à fait entendre un nouveau discours, jusque-là trop absent de notre littérature. Bilan: L'événement central de la production littéraire des années soixante-dix réside dans cette multiplicité des écritures, des voix, j'appelle cet événement la naissance de la polyphonie québécoise (p. 123). 
Bien entendu, si je voulais faire le pion, je pourrais mettre en question la notion de modernité utilisée par Haeck. En gros, pour lui, modernité est synonyme de vie, de nouveauté, d'anti-tradition. Ce qui, en un sens, n'est pas faux mais demeure bien général. J'en veux pour preuve que dans son dernier texte, "certitudes touchées", il fait remonter la modernité québécoise tantôt à Carquois d'Albert Pelletier, tantôt à un texte du frère Marie-Victorin (1925), tantôt à un article de Jules Fournier (1914). Sur le plan théorique il la rattache à la constellation Marx-Nietzsche-Freud, sur le plan esthétique à Baudelaire, ce qui encore là n'est pas faux mais demeure vague. On sait qu'Habermas la fait remonter au siècle des Lumières, d'autres à la Renaissance. Tout cela est vrai d'une certaine manière compte tenu des définitions qu'on en propose. Mais justement la définition de Haeck n'est pas claire, c'est le moins qu'on puisse dire: comme d'autres, cette notion lui sert un peu de concept passe-partout et partant, à mon sens, n'éclaire pas grand chose.

Ceci dit il s'agit là d'une faiblesse vraiment mineure d'un ouvrage au contenu très riche dans l'ensemble. Haeck réussit en effet à être à la fois savant et personnel, distant (lucide) et engagé. Il propose un regard informé et neuf sur la production littéraire québécoise des dernières années dont il a été un artisan et non des moindres. Cette production, visiblement, le passionne et il en parle avec générosité et intelligence, dans une sorte de complicité non complaisante avec les écrivains qu'il évoque. Que demander de plus à un livre dont la lecture est une réjouissance pour la tête et le cœur?

1. Philippe Haeck, la Table d'écriture. Poétique et modernité, Montréal, VLB, éditeur, 1984, 386 p. 